\title{
Rate-Flexible Optical CDMA Networks based on Coherent Modulation Formats
}

\author{
Anderson Sanches, ${ }^{1}$ Member, IEEE, Mohamed Abuhelala ${ }^{2}$, Student Member, IEEE, Thiago R. Raddo, ${ }^{1}$ \\ Member, IEEE, Shyqyri Haxha ${ }^{3}$, Senior Member, IEEE, and Ivan Glesk ${ }^{2}$, Senior Member, IEEE \\ ${ }^{I}$ Engineering, Modeling, and Applied Social Sciences Center, Federal University of ABC, Santo Andre, Brazil \\ ${ }^{2}$ Faculty of Engineering, University of Strathclyde, Glasgow, United Kingdom \\ ${ }^{3}$ Department of Electronic Engineering, Royal Holloway, University of London, United Kingdom \\ anderson.sanches@ufabc.edu.br
}

\begin{abstract}
In this paper, we propose, design, and assess the performance of optical coherent access networks based on optical code-division multiple-access (OCDMA) based on three distinct coherent modulation formats, namely, BPSK, QPSK, and 16-QAM. We design a flexible network architecture based on these coherent modulation formats to provide either single data rate transmissions or multiple data rate transmissions. In this way, the network supports users with the same transmission rate, or group of users transmitting at different data rates.

We derive new close-form analytical expressions for both single and multiple rate use cases to assess the performance of the coherent OCDMA network. Results shown that the multi-rate data traffic from the users can coexist almost transparently in terms of network performance. The flexible OCDMA network might be a prospective alternative for implementing coherent systems for different service provision according to traffic demand requirements while supporting with high capacity, security, and simultaneous numbers of subscribers. Keywords: BPSK, QPSK, 16-QAM, coherent network, BER, OCDMA
\end{abstract}

\section{INTRODUCTION}

The recent advance on optical coherent technology is leading coherent networks to new and unexplored use cases and markets [1]. This new technology trend is partly due to the progress on photonic integrated circuit (PIC) technology [2]. able to satisfy energy consumption, massive fabrication and cost requirements for optical coherent transceivers [3] including compact form factor broadband laser sources as optical frequency comb generator [4]. Coherent networks are normally employed for long-reach distances such as metro- and long-haul transmissions covering over thousands of kilometers [5]. Despite it, coherent optical networks are gaining relevance as well as market share also for short- and medium-reach distances such as data center and passive optical network (PON) [6]. The optical maturity in coherent devices have broadened the coherent PON's reach into end-user business premises and even homes, allowing new services to be delivered to customers. This eventual paradigm shift and technological trend, will open new opportunities while trying to satisfy challenging requirements such as spectral efficiency, optical filtering, and security in PON.

Coherent techniques have become attractive also due to high capacity over non-coherent systems, which can support future insatiable growing data traffic demand and still offer flexibility in architecture design, becoming of general interest also for access networks. To this aim, optical coherent technologies are perceived here as a prospective solution to be implemented along with code-division multiple-access (OCDMA) multiplexing technique for broadband service provision in PON. By its turn, OCDMA has unique inherent features, like, for example, asynchronism access to the transmission channel (which makes this technique particularly useful and efficient for practical network deployment), higher security support, and soft multiplexing, to name but a few [7]-[12].

In this paper, we propose, design, and investigate the performance of coherent optical access networks based on OCDMA multiplexing technique. We design a flexible network architecture based on coherent modulation formats that can operate and provide either as single data rate transmissions or multiple data rate transmissions. In this manner, network's users can all have the same transmission rate or a group of users can have higher data rates. This is particularly interesting for different network service provision according to distinct traffic demand requirements. To do so, optical coherent modulation formats such as binary phase-shift keying (BPSK), quadrature binary phase-shift keying (QPSK), and 16 quadrature amplitude modulation (16-QAM) are considered during the network design. OCDMA allows for a more efficient and flexible management of the network resources based on optical codes whereas coherent high-order modulation formats and high symbol rates allows for large system capacity. For example, QPSK users can achieve twice the data rates of BPSK users whereas 16-QAM users can achieve twice the data rates of QPSK users.

We derive new close-form analytical expressions for both single and multiple rate provision in order to evaluate the performance of the coherent OCDMA network. 
We carry out a performance analysis of a single rate service network, where all users transmission at the same data rate. Then, we also investigate a multiple rate service network considering different groups of users, where each group transmits at its own data rate.

Analytical results have shown that users can transmit simultaneously information at different data rates and similar network performances. Hence, coherent multi-rate OCDMA networks are good technology candidates for flexible implementation of NG-PON2 with high capacity, security, and number of users.

\section{OPTICAL COHERENT NETWORK DESCRIPTION}

We design a rate-flexible OCDMA network in a star configuration capable of proving single-rate and multiplerate transmissions through different coherent modulation formats [8]. The rate-flexible OCDMA network can employ three distinct modulation formats, i.e., BPSK, QPSK, and 16-QAM according to the user group rate requirements. In this way, low transmission users employ BPSK whereas high transmission users employ 16QAM. The block diagram of the proposed rate-flexible OCDMA network is shown in Figure 1.

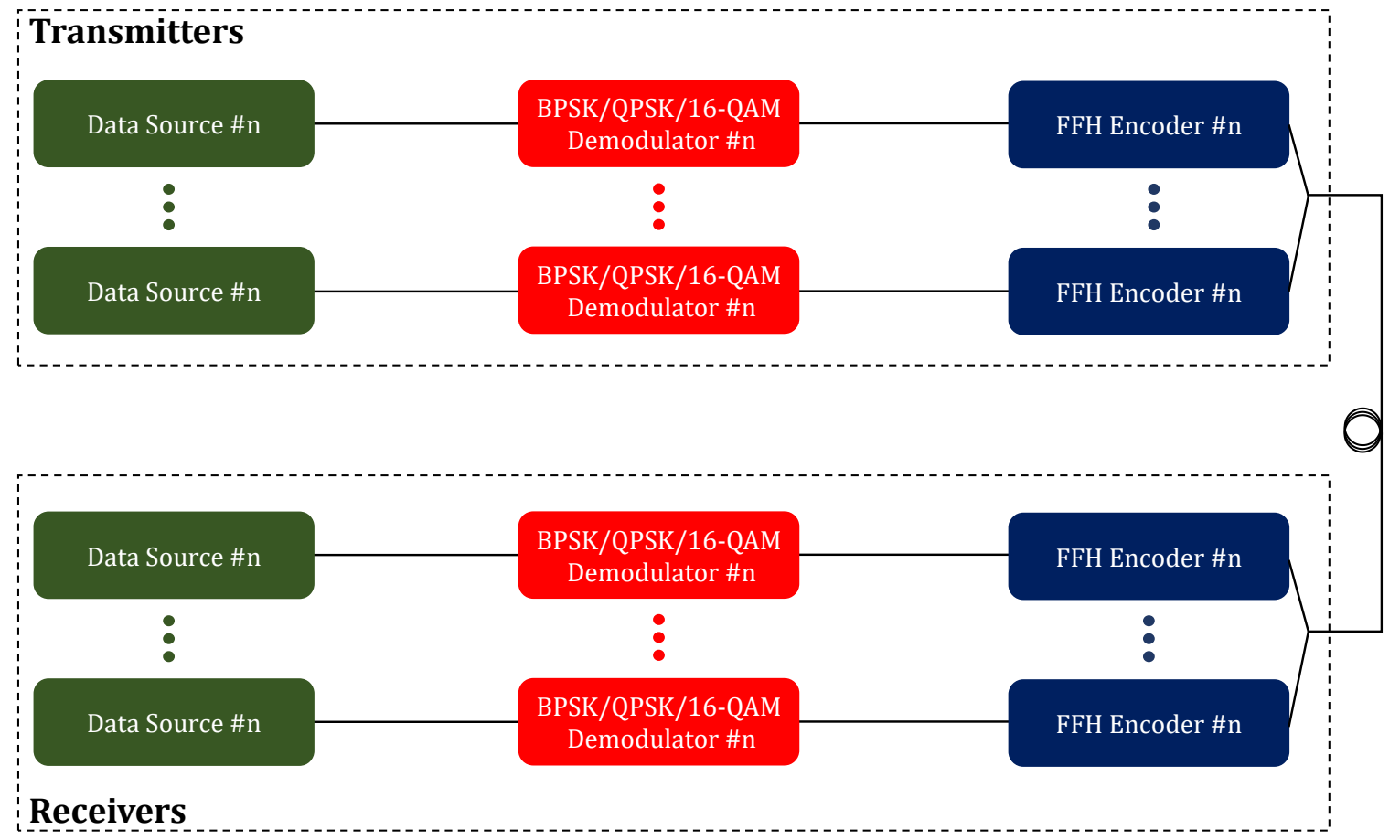

Figure 1.Block diagram of the proposed rate-flexible OCDMA network.

Now, it is assumed that MAI is the main degrading factor of the network while ignoring other noise sources and that decision error occurs when the desired user transmits a data bit " 0 " and " 1 " [8]. For convenience sake, only closed-form equations for 16-QAM is derived here. In this case, the variance associated with highrate users is the same as for the 16-QAM modulation format.

Besides, it is necessary to express the variance for the cases where the interfering users of a given group are demodulated by the receiver of the desired user that is active in another class. Therefore, consider first that the interfering users transmit data bits at other rates than high-rate class and their signals are demodulated in the receptor of the desired user, which is designed for high-rates. At the receiver's side, assuming interfering active users transmitting signals in the network, the received optical field at the decoder output of the desired user will be

$$
E_{16-Q A M_{n}}^{I, Q}\left(t, \theta_{b}\right)=E_{a c_{-} 16-Q A M_{n}}^{I, Q}\left(t, \theta_{b}\right)+\sum_{k=1}^{K} E_{\left.c c_{-} \neq n\right)}^{I, Q} 16-Q A M_{n, k}\left(t-\tau_{k}, \theta_{b}\right),
$$

where $I, Q$ denotes the in- and quadrature-phase channels, respectively, the $E_{a c_{-} 16-\mathrm{QAM}_{n}}$ is the autocorrelation signal at the desired user $n, E_{c c_{-} 16-\mathrm{QAM}_{n, k}}$ is the cross-correlation signal between the desired user $n$ and the interfering user $k, \theta_{b}$ is the phase associated to the information bit and $\tau_{k}$ is the chip delay for the $k$ interfering user. 
The BER formalism explicitly requires the decision variables of the desired user to be defined in terms of auto- and cross-correlation signals. In this context, the average level of the desired signal can be written as

$$
\mu_{16-Q A M_{n}}^{I, Q}=\mu_{16-Q A M_{a c}}^{I, Q}+\mu_{16-Q A M_{M A I}}^{I, Q}
$$

with $\mu_{16-Q A M_{a c}}^{I, Q}$ and $\mu_{16-Q A M_{M A I}}^{I, Q}$ representing the contributions of the autocorrelation and cross-correlation signals to decision variable, respectively.

Once the interfering users are statistically independent, it is convenient to express the cross-correlation signal level when only two interfering users transmit simultaneously. Thus, the instantaneous MAI power in the $j$ interfering user's receiver when the $k$ interfering user transmits data in I and Q channels are given, respectively, by

$$
\begin{aligned}
\mu_{16-Q A M_{M A I_{j, k}}^{I, Q}}^{I,} & =\mathcal{R} e\left\{\frac{\Re}{4} \times\left[\int _ { T _ { C a } } \left(E_{c c_{-} 16-Q A M_{j, k}}{ }^{*}\left(t-\tau_{k}, \theta_{b}\right) E_{L O_{n}}^{I, Q}(t)\right.\right.\right. \\
& \left.\left.\left.+E_{c c_{-} 16-Q A M_{j, k}}\left(t-\tau_{k}, \theta_{b}\right) E_{L O_{n}}^{I, Q}{ }^{*}(t)\right) d t\right]\right\},
\end{aligned}
$$

where $E_{L O_{n}}^{I, Q}$ is the local oscillator signal, $\mathfrak{R}$ is the photodetector's responsivity and * denotes the complex conjugate operator. First, since it is not known which coded symbols will be active at any given time, an average calculation $\overline{\left(\mu_{16-\mathrm{QAM}_{M A I} I, \mathrm{Q}}^{I, Q}\right.}$ ) considering all possible coded symbols and random-access delay of every user is required. Therefore, the interference variance becomes

$$
\sigma_{16-Q A M_{M A I_{j, k}}^{2}}^{I, Q}=\left(\mu_{16-Q A M_{M A I_{j, k}}^{I, Q}}-\overline{\mu_{16-Q A M_{M A I_{j, k}}^{I, Q}}}\right)^{2} .
$$

Finally, the minimal BER for 16-QAM based networks can be approximated to the probability of error of a symbol which is not in the corner or not in the inside the constellation diagram [13]

$$
B E R_{16-Q A M} \approx 1-\left[\left(1-\frac{1}{2} B E R_{16-Q A M}^{I}\right)\left(1-B E R_{16-Q A M}^{Q}\right)\right]
$$

where

$$
B E R_{16-Q A M}^{I, Q}=Q\left(\sqrt{S I R_{16-Q A M}^{I, Q}}\right)
$$

and

$$
S I R_{16-Q A M_{-} M R}^{I, Q}=\frac{\left(\mu_{16-Q A M_{a c}}^{I, Q}\right)^{2}}{K_{h r} \overline{\sigma_{16-Q A M_{M A I, h r}}^{2}+Q} K_{\text {other }} \overline{\sigma_{\text {other }}^{2}}}
$$

\section{SIMULATION RESULTS}

In this section, the previously described formalism is now applied to the analysis of a rate-flexible OCDMA network based on BPSK, QPSK and 16-QAM modulation formats. Further, it is evaluated the performance analysis in the limit case where all users transmit data at identical modulation format and the scenarios converge towards a conventional or single-rate operation. At this point, it is worth to point out the common parameters adopted for both single-rate and rate-flexible operations. Then, the base functions used to generate the $I$ and $Q$ carriers for the QPSK and 16-QAM symbols have the same optical field amplitude relative to this one employed in BPSK symbol generation. Such consideration implies to make the optical symbol amplitude higher by $\sqrt{2}$ and $3 \sqrt{2}$ for QPSK and 16-QAM transmissions, respectively. This situation leads to a higher interferent scenario when compared to the case where the average power per symbol is maintained constant regardless of the modulating format adopted. In addition, it is assumed that the users transmit data under synchronization, a situation that does not take the advantage of asynchronous traffic in OCDMA networks, 
but greatly simplifies the formalism and the BER equations represent the worst case for the network performance.

Furthermore, the OCDMA encoders produce a set of FFH signature sequences is composed of 29 codes holding 12 chips each. Within a specific sequence, each chip utilizes one of the 29 optical carriers at a specific wavelength chosen among the 29 wavelengths available in the range of $1530 \mathrm{~nm}-1630,8 \mathrm{~nm}(3,6 \mathrm{~nm}$ spacing). The OCDMA encoders/decoders modeled as Bragg gratings with a sinc apodization profile allow obtaining nearly disjoint and high-density wavelength slices. It is possible by using devices with a high modulation index depth of the order of $2.6 \times 10^{-3}$ with and $1 \mathrm{~mm}$ long. At the receiver side, the OCDMA decoders act as a matched filter and remove the wavelength translation introduced in the encoder.

Consequently, the desired user signal arrives as a high autocorrelation pulse and all the interfering signals that do not coincide in time and wavelength are manifested as cross-correlation peaks. Finally, the coherent demodulation is carried out by mixing the decoded signal with a broadband pulse replica of the autocorrelation signal provided by the local oscillator (LO).

Also, the transmission data rates chosen were fixed in $10 \mathrm{Gbaud} / \mathrm{ps}$ for both kinds of networks. Consequently, transmission data bit rates adopted for the rate-flexible network were set in 10, 20 and $40 \mathrm{~Gb} / \mathrm{s}$ for the first-, second- and third-classes, respectively. On the other hand, the transmission data bit rate was set in 10, 20 and $40 \mathrm{~Gb} / \mathrm{s}$ for BPSK-, QPSK- and 16-QAM-based networks. It is worth mentioning that these transmission data rates are compatible with the next generation of PON.

Once all common network configuration parameters are properly described, the performance associated with the networks can now be discussed.

Initially, it is interesting to analyze the performance of the single-rate network. The results of the BER as a function of the simultaneous users are shown in Figure 2. As can be observed in this Figure, BPSK-based network can support up to 23 simultaneous users whereas both the QPSK- and 16-QAM-based networks support 22 simultaneous users under an error-free environment. The similar performance of these independent networks is quite interesting by suggesting a similar behavior if such modulating formats are used to compose a multi-rate network. It should be remembered that conventional OOK-based multi-rate FFH-OCDMA networks utilize different code lengths to users' signatures and, therefore, have the performance of high-class users severely degraded.

Next, the performance of the proposed coherent multi-rate OCDMA network is investigated. The results of the BER as a function of the simultaneous users are shown in Figure 2.

In this case, the set of 29 codes available is assigned to a maximum number of 10 and 9 simultaneous users for both the first and second classes, and 9 for the third-class network, respectively. As expected, the results confirm the aforementioned guess and show that the network performance of users in different classes is similar. Moreover, performance analysis reveals that the maximums of 24 (5 users at desired low-rate class, 10 users at the middle-rate class and 9 users at the high-rate class), 23 (10 users at desired low-rate class, 4 users at the middle-rate class and 9 users at the high-rate class) and 22 (10 users at desired low-rate class, 10 users at the middle-rate class and 2 users at the high-rate class) simultaneous users can coexist with BER levels maintained below the error-free limit when the desired user transmits data at low-, middle- and highclass, respectively.

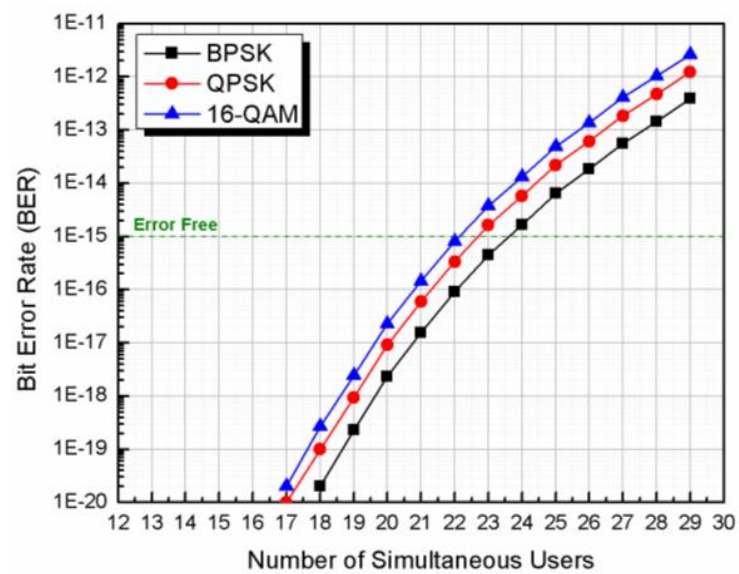

(a)

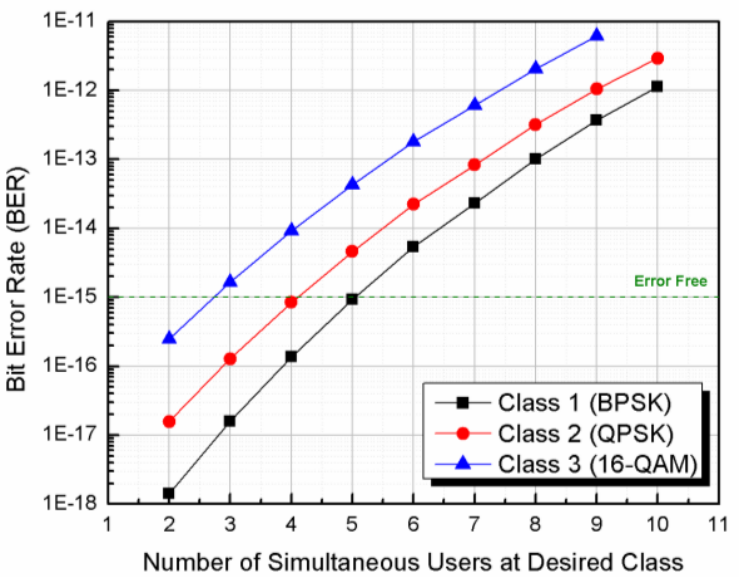

(b)

Figure 2.BER performance of the flexible-rate coherent OCDMA network. (a) single-rate use case: all users transmit at the same rate. (b) multiple-rate use case: user groups transmit at different rates according to the modulation format. 


\section{CONCLUSIONS}

In this paper, we have proposed a new OCDMA network architecture based on coherent modulation formats such as BPSQ, QPSK, and 16 QAM. OCDMA allows for a more efficient and flexible management of the network resources based on optical codes whereas coherent high-order modulation formats and high symbol rates grant the system larger capacity. This technology combination is key to cope with growing data traffic demand while allowing the design of a flexible network. The network architecture is flexible in a way that allows the deployment of just a single modulation format or the combination of the three formats concurrently. The latter, allows users of the network to achieve different data rate transmissions, which can be especially useful for different data traffic requirements.

Furthermore, we have derived new close-form analytical expressions for both coherent network user cases in order to assess the performance of the system. It is worth mentioning that these expressions can be further useful and straightforwardly adapted for coherent networks based not only on different OCDMA coding schemes, but also based on distinct multiplexing techniques such as TDMA, WDMA, and OFDM. Moreover, we carried out a performance analysis of a single rate network with all users transmitting at the same rate, and also of a multiple rate network with different data rate groups of users. In the latter network, each user group transmits at its own data rate. The results revealed that users can achieve similar BER performance for users' classes operating in very distinct data rates. Thus, the PSK modulation formats shown to be a robust for implementation in OCDMA networks. Accordingly, coherent multi-rate OCDMA networks might be a prospective technology alternative for flexible implementation of NG-PON2 with higher capacity, security, and simultaneous number of subscribers.

\section{ACKNOWLEDGEMENTS}

This work was partially supported by the Sao Paulo Research Foundation (FAPESP) under grant number 2017/26338-9.

\section{REFERENCES}

[1] Han Sun et al. "800G DSP ASIC design using probabilistic shaping and digital sub-carrier multiplexing", IEEE/OSA JLT 2020.

[2] M. K. Smit and K. A. Williams, "Indium Phosphide Photonic Integrated Circuits," in Optical Fiber Communication Conference (OFC) 2020, OSA Technical Digest (Optical Society of America, 2020), paper W3F.4.

[3] V. Lal et al., "1.6Tbps Coherent 2-Channel Transceiver using a Monolithic Tx/Rx InP PIC and Single SiGe ASIC," OFC 2020.

[4] Pablo Marin-Palomo, Juned N. Kemal, Tobias J. Kippenberg, Wolfgang Freude, Sebastian Randel, and Christian Koos, "Performance of chip-scale optical frequency comb generators in coherent WDM communications," Opt. Express 28, 2020.

[5] A. Nespola et al., "Transmission of $61 \mathrm{C}$-band Channels with L-band Interferers over Record 618km of Hollow-Core-Fiber," in Optical Fiber Communication Conference Postdeadline Papers 2020, (Optical Society of America, 2020), paper Th4B.5.

[6] D. van Veen, "Transceiver Technologies for Next-Generation PON (Tutorial)," in OFC 2020.

[7] H. Mrabet, A. Cherifi, T. Raddo, I. Dayoub and S. Haxha, "A Comparative Study of Asynchronous and Synchronous OCDMA Systems," in IEEE Systems Journal, 2020.

[8] A. L. Sanches, T. R. Raddo, J. V. dos Reis, and B.-H. V. Borges, "Performance analysis of single and multirate FFH-OCDMA networks based on PSK modulation formats," IEEE/OSA J. Opt. Commun. Netw., vol. 7, no. 11, pp. 1084-1097, Nov. 2015.

[9] T. R. Raddo, A. L. Sanches, I. T. Monroy, and B.-H. V. Borges, "Throughput performance evaluation of multiservice multirate OCDMA in flexible networks," IEEE Photon. J., vol. 8, no. 1, Feb. 2016

[10] J.V. dos Reis, T.R. Raddo, A.L. Sanches, and B.-H.V Borges, "Fuzzy Logic Control for the Mitigation of Environmental Temperature Variations in OCDMA Networks," to appear in IEEE/OSA J. Opt. Commun. and Netw., 2015.

[11] C. Chang, G. Yang, I. Glesk and W. C. Kwong, "On The Performance of The Effects of Temperature Variation in Ultrafast Incoherent Fiber-Optic CDMA Systems With SOA-Based Tunable Dispersion Compensator," in IEEE Photonics Journal, vol. 11, no. 3, pp. 1-10, June 2019.

[12] T. Kodama and G. Cincotti "OCDM systems with PSK and QAM codes", Optics Communications, vol. 465, 125538, June 2020.

[13] J. R. Barry, E. A. Lee, and D. G. Messerschmitt, Digital Communications, 3rd ed. Norwell, MA: Kluwer, 2004. 